\title{
Optical Coherence Tomography Angiography Offers New Insights into Choriocapillaris Perfusion
}

\author{
Jost L. Lauermann Nicole Eter Florian Alten \\ Department of Ophthalmology, University of Münster Medical Center, Münster, Germany
}

\section{Keywords}

Optical coherence tomography - Optical coherence tomography angiography $\cdot$ Spectral-domain optical coherence tomography. Swept-source optical coherence tomography · Choriocapillaris · Age-related macular degeneration $\cdot$ Central serous chorioretinopathy

\begin{abstract}
The choriocapillaris (CC) represents a fundamentally important vascular layer that is subject to physiologic changes with increasing age and that is also associated with a wide range of chorioretinal diseases. So far, information on blood flow in this specific layer has remained limited. With the advent of optical coherence tomography angiography (OCTA), new perspectives and possibilities of CC imaging have begun to evolve. This article shall review the opportunities and challenges of applying OCTA technology to the CC layer and summarize the current clinical efforts in OCTA CC imaging exemplarily in dry age-related macular degeneration and central serous chorioretinopathy.

(c) 2018 S. Karger AG, Basel
\end{abstract}

\section{Introduction}

The choriocapillaris (CC) represents an approximately $10-\mu \mathrm{m}$-thin layer of relatively large-diameter capillaries interconnected in a densely packed arrangement with very small intercapillary pillars located at the inner aspect of the choroid $[1,2]$. It shares a mutualistic relationship with the retinal pigment epithelium (RPE). If one is compromised, the other will follow, and eventually both layers may degenerate within a short time [3]. Changes in $\mathrm{CC}$ are known to occur physiologically with increasing age and to be associated with a wide range of retinal diseases such as age-related macular degeneration (AMD) and central serous chorioretinopathy (CSC), which are major causes of vision loss [4-8]. Thus, visualization and quantification of CC microvasculature and CC blood flow are of great interest.

Due to the two-dimensionality of conventional fluorescein angiography (FA) and indocyanine green angiography (ICGA), it has so far not been possible to attribute angiographic findings specifically to the different retinal and choroidal layers. ICGA cannot visualize the CC separately from the layers of the medium and large vessels of the choroid. In addition to the lack of depth selectivity, imaging CC using angiography is also challenging due to dye leakage and its location behind the light-absorbing

\section{KARGER}

(c) 2018 S. Karger AG, Basel

E-Mail karger@karger.com

www.karger.com/oph
Jost L. Lauermann, MD

Department of Ophthalmology, University of Münster Medical Center Domagkstrasse 15

DE-48149 Münster (Germany)

E-Mail jost.lauermann@ukmuenster.de 
Table 1. Comparison of five different OCTA devices

\begin{tabular}{|c|c|c|c|c|c|}
\hline Device & $\begin{array}{l}\text { OCT } \\
\text { technology }\end{array}$ & Algorithm & $\begin{array}{l}\text { Light source and } \\
\text { scanning speed }\end{array}$ & $\begin{array}{l}\text { Choriocapillaris } \\
\text { slab }\end{array}$ & $\begin{array}{l}\text { FDA } \\
\text { approval }\end{array}$ \\
\hline Optovue AngioVue & SD-OCT & SSADA & $\begin{array}{l}840 \mathrm{~nm} \text { wavelength, } \\
70,000 \mathrm{~A}-\mathrm{scan} / \mathrm{s}\end{array}$ & $\begin{array}{l}30-60 \mu \mathrm{m} \text { below } \\
\text { the RPE }\end{array}$ & yes \\
\hline $\begin{array}{l}\text { Heidelberg Spectralis } \\
\text { OCTA }\end{array}$ & SD-OCT & FSADA & $\begin{array}{l}870 \mathrm{~nm} \text { wavelength, } \\
85,000 \mathrm{~A} \text {-scan/s }\end{array}$ & manual segmentation & no \\
\hline Canon OCT-HS100 & SD-OCT & modified FSADA & $\begin{array}{l}855 \mathrm{~nm} \text { wavelength, } \\
70,000 \mathrm{~A} \text {-scan/s }\end{array}$ & $\begin{array}{l}0-20 \mu \mathrm{m} \text { below } \\
\text { Bruch's membrane }\end{array}$ & no \\
\hline Zeiss Angioplex & SD-OCT & OMAG & $\begin{array}{l}840 \mathrm{~nm} \text { wavelength, } \\
68,000 \mathrm{~A}-\mathrm{scan} / \mathrm{s}\end{array}$ & $\begin{array}{l}29-49 \mu \mathrm{m} \text { below } \\
\text { the RPE }\end{array}$ & yes \\
\hline Zeiss PLEX Elite 9000 & SS-OCT & OMAG & $\begin{array}{l}1,050 \mathrm{~nm} \text { wavelength, } \\
100,000 \mathrm{~A}-\mathrm{scan} / \mathrm{s}\end{array}$ & $\begin{array}{l}29-49 \mu \mathrm{m} \text { below } \\
\text { the RPE }\end{array}$ & yes \\
\hline Topcon SS-OCTA & SS-OCT & OCTARA & $\begin{array}{l}1,050 \mathrm{~nm} \text { wavelength, } \\
100,000 \mathrm{~A}-\mathrm{scan} / \mathrm{s}\end{array}$ & $\begin{array}{l}0-10.4 \mu \mathrm{m} \text { below } \\
\text { Bruch's membrane }\end{array}$ & no \\
\hline
\end{tabular}

FDA, Food and Drug Administration; FSADA, full-spectrum amplitude decorrelation algorithm; OCT, optical coherence tomography; OCTA, OCT angiography; OCTARA, OCT angiography ratio analysis; OMAG, OCT-based optical microangiography; RPE, retinal pigment epithelium; SD-OCT, spectral-domain OCT; SSADA, split-spectrum amplitude-decorrelation angiography; SS-OCT, swept-source OCT.

RPE. The lack of an imaging modality capable of visualizing and quantifying CC blood flow in vivo has so far hindered a better understanding of various chorioretinal diseases. As a result, pathophysiologic theories concerning the role of CC blood flow are still primarily based on histologic findings and studies using structural optical coherence tomography (OCT).

OCT angiography (OCTA) represents a novel noninvasive imaging tool that allows for the first time a selective in vivo CC visualization in humans as well as in animal models $[9,10]$. The aim of this article is to review the possibilities and challenges of using OCTA technology in CC imaging and to summarize current clinical efforts of applying OCTA CC imaging exemplarily in dry AMD and CSC.

\section{Technical Considerations}

Although OCTA is still a novel imaging technique, various manufacturers already offer commercially available OCTA devices. The predominant devices on the market are AngioVue ${ }^{\mathrm{TM}}$ (Optovue Inc., Fremont, CA, USA), Angioplex ${ }^{\circledR}$ and PLEX ${ }^{\circledR}$ Elite 9000 (Carl Zeiss Meditec Inc., Dublin, CA, USA), Swept-Source Optical Coherence Tomography Angio ${ }^{\mathrm{TM}}$ (Topcon Corp., Japan),
Heidelberg Spectralis ${ }^{\circledR}$ OCTA (Heidelberg, Germany), and Canon OCT-HS100 (Canon, Japan). With the exception of the Topcon device, all devices have been tested in clinical routine at our clinic. All devices show a variety of technical differences in hardware and software (Table 1; Fig. 1). OCTA calculates the differences between B-scans and is based on the assumption that differences between $\mathrm{B}$-scans arise from blood flow and that the backscattering from retinal tissue outside the vessels remains static. There are two ways to detect change in amplitude between B-scans: (1) speckle (or intensity) decorrelation, which detects intensity changes in OCT structural images, and (2) phase variance, which assesses changes in the phase of a light wave. Based on the signals, OCTA can be full-spectrum or split-spectrum.

Different software algorithms are used to realize visualization and quantitative analysis such as OMAG (OCTbased optical microangiography), SSADA (split-spectrum amplitude-decorrelation angiography), or OCTARA (OCT angiography ratio analysis) [11, 12]. A comparison of two OCTA devices with different software approaches (SSADA and OMAG) with focus on CC quantification measurements resulted in a basic agreement between measurements [13]. However, the results of different devices are currently not transferable. When comparing OCTA imaging of different devices, device-specif- 

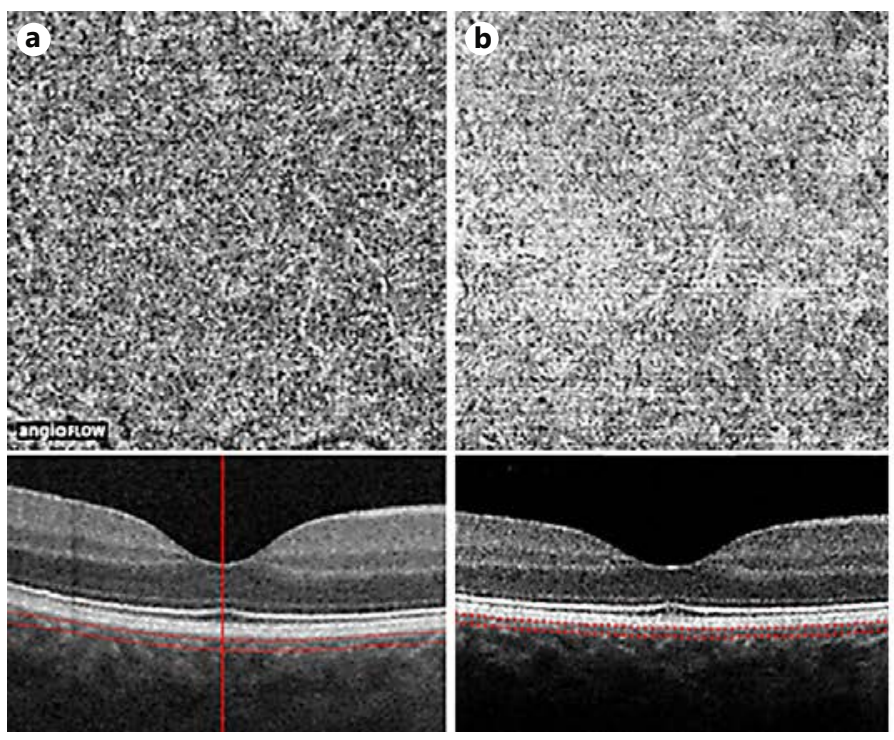

Fig. 1. Optical coherence tomography angiography (OCTA) choriocapillaris $(\mathrm{CC})$ en face images with corresponding B-scan below of a healthy control subject ( 28 years, female) taken by four different devices of different manufacturers. a Optovue AngioVue, spectral-domain OCTA, CC slab thickness 30-60 $\mu \mathrm{m}$ below the retinal pigment epithelium (RPE) reference. b Heidelberg Spectra-

ic characteristics must always be considered. More comparative CC studies including more different devices are needed in this context.

Looking at the hardware, the use of two different key OCT technologies represents one major difference between current devices. While most devices are based on spectral-domain OCT (SD-OCT) (wavelength 840-870 $\mathrm{nm}$ ), two are equipped with swept-source OCT (SS-OCT) technology (wavelength 1,050 nm) (Table 1). SD-OCT works with a near-infrared light source and a spectrometer as a detector, SS-OCT uses a swept laser and a single photodiode detector $[14,15]$. At first sight, SS-OCT appears as the more advantageous technology for imaging deeper structures underneath the RPE due to its longer wavelength, reduced sensitivity roll-off, and higher scanning speeds. Interestingly, retina specialists generally seem to prefer SS-OCT to SD-OCT, as recently reported using the example of the two Zeiss OCTA devices Angioplex and PLEX Elite 9000. Although most physicians preferred the SS-OCTA to the SD-OCTA device, $61 \%$ of the participants stated that both devices were equally valuable [16]. So far, the high cost of the SS-OCT technology has presumably limited widespread commercial and clinical acceptance of this method to date. Notably, large studies on the superiority of SD-OCTA or SS-OCTA in
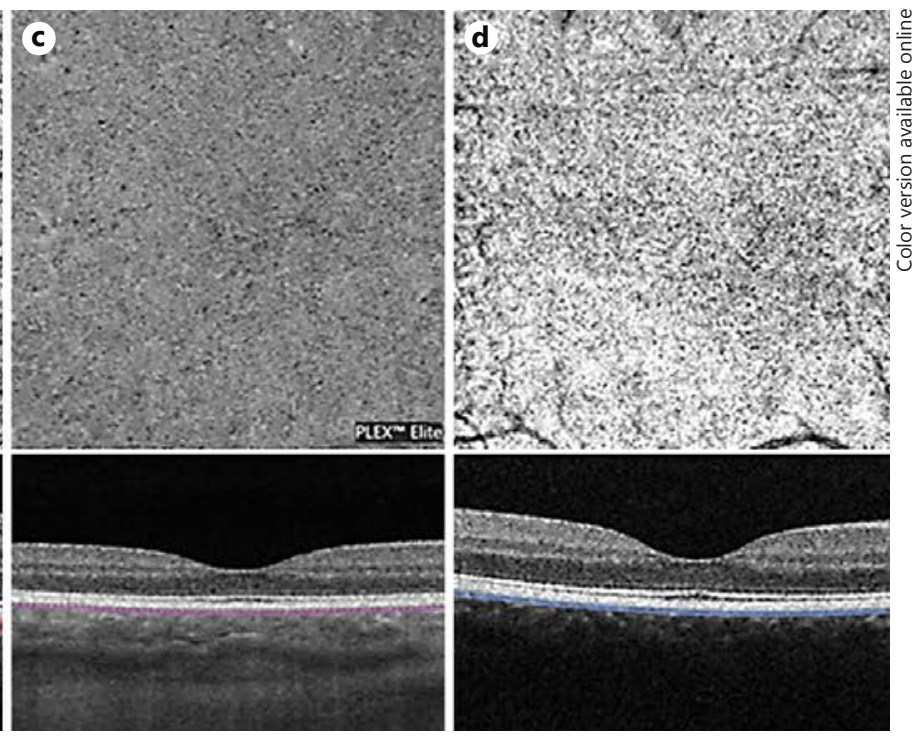

lis OCTA, spectral-domain OCTA, slab thickness 29-49 $\mu \mathrm{m}$ below the RPE reference (manual segmentation, no presetting by the manufacturer). c Zeiss PLEX Elite 9000, swept-source OCTA, slab thickness 29-49 $\mu \mathrm{m}$ below the RPE reference. d Canon OCTHS100, slab thickness 0-20 $\mu \mathrm{m}$ below Bruch's membrane reference.

quantifying CC blood flow and detecting pathophysiologic changes in the CC layer are still missing.

Regardless of the device, image artifacts are a major limitation of OCTA imaging. Fortunately, many issues are already being addressed as OCTA technology quickly moves forward. In general, challenges in image quality in OCTA can result from motion, segmentation errors, projection, or anatomical limitations such as shadowing of the vitreous body. While the latter is difficult to overcome technically, the first three ones are being worked on with the first technical approaches already published.

(1) OCTA is a motion contrast technique based on changes in successively recorded images. Motion between repeated images can be caused by axial and transverse eye motion causing various types of artifacts [17, 18]. Software algorithms can compensate for some movement artifacts, but can also produce artifacts themselves. Particularly in patients with macular diseases, fixation and vision are often significantly reduced, resulting in a higher potential of creating motion artifacts. A recent study proved the high benefit of eye tracking in OCTA imaging in AMD patients regarding the expression of motion artifacts [19]. Meanwhile, most commercial OCTA devices have been equipped with an eye tracking system, which also warrants improved intrasession re- 
peatability as well as reliable follow-up examinations over time.

(2) Segmentation errors in OCTA are frequent in the presence of retinal pathologies and can affect all layers, including the CC [20]. Notably, en face imaging requires an accurate segmentation of retinal layers, which can be reliably achieved in normal maculae. However, pathologic changes may disturb the assumptions made by automated software segmentation algorithms. Most segmentation methods of retinal layers are based on intensity information. The boundaries of the internal limiting membrane (ILM) and the RPE are the most prominent ones in SD-OCT images because their intensity contrast is usually highest [21]. Therefore, the ILM and the RPE usually allow for robust segmentation, whereas the segmentation of layers between the ILM and the RPE is more complex and segmentation success is more limited by intensity discontinuity and inconsistencies in the retinal layers.

A reliable segmentation of CC in early and intermediate AMD was demonstrated in a recent OCTA study [22]. However, fully automated CC segmentation may pose a problem, e.g., in larger pigment epithelium detachment (PED) [23]. It appears as if the higher the PED lesion, the less reliable the detection of the correct segmentation plane and the adherence to this plane in all sections. The general applicability of OCTA to vascularized PED lesions has been demonstrated by Veronese et al. [24] using semi-automated segmentation with manual correction of the CC layer. In addition to the dome shape of PED, the ultrastructure of the sub-RPE choroidal neovascular membrane can also disturb CC segmentation. Particularly in fibrovascular PED lesions, a certain architecture of several layers can be observed, with neovascular vessels and fibrotic tissue misleading the segmentation algorithm [25]. This challenge of CC segmentation also applies to PED in CSC, large drusen in early and intermediate AMD, or geographic atrophy (GA). However, no data on these entities are available yet. More elaborate and robust software algorithms for automated CC segmentation in pathologically altered retinas are required.

(3) Projection artifacts have a high relevance in imaging CC. Structures from the overlying retinal vascular architecture can falsely appear on the level of the CC. The definition of the CC slab has high relevance regarding this effect. If the CC slab is positioned at the expected anatomical level, the image is dominated by retinal vascular projection. Interestingly, the $\mathrm{CC}$ can be more reliably visualized by choosing a slab slightly posterior to the anatomically correct location of the CC [26]. The CC flow produces OCTA decorrelation signals that are persistent at depths posterior to the anatomic CC called decorrelation tails or shadowing. Erythrocytes are extremely scattering and produce fluctuations in the OCT beam below them, causing deeper structures to exhibit variations in the OCT signal. This can produce artifacts, but allows for a more robust visualization of vasculature [26]. The exact localization and thickness of the CC slab vary between 0 and $34 \mu \mathrm{m}$ below the RPE/Bruch's membrane reference and between 4.4 and $30 \mu \mathrm{m}$, respectively, depending on the device used [17, 20, 27, 28]. Notably, manufacturers use various definitions of the CC slab level, and not all of them offer a presetting for CC examination yet (Table 1 ).

Another challenging aspect in OCTA CC imaging is that CC flow is slow compared to blood flow in retinal vessels. In rats, the velocity of blood flow was found to be four times slower in the CC than in the inner retinal capillaries [29]. In current OCTA imaging technology, there is a slowest detectable flow, which is determined by the elapsed time between consecutive cross-sectional scans, approximately $<0.3 \mathrm{~mm} / \mathrm{s}$. There is also a fastest detectable flow, or saturation limit, when vascular flows above a given value will produce the same decorrelation signal. The CC flow may occasionally be too slow, resulting in a signal void. The decorrelation signal can be more sensitive to slower flows if the time between consecutive crosssectional scans is increased. However, by doing so, OCTA also becomes more sensitive to eye motion, which increases artifact image noise [30]. An interesting approach is the variable interscan time analysis (VISTA) algorithm which was firstly described by Choi and colleagues [27, 31], a tool that allows for distinguishing varying degrees of CC flow impairment. Notably, this method provides a relative blood flow measurement and is only applicable to slow flows of $<0.3 \mathrm{~mm} / \mathrm{s}$.

Current OCTA devices do not offer an automatic quantification of CC flow and technically, absolute quantification of CC flow remains challenging for several reasons. Nevertheless, different approaches for CC flow quantification have been used in previous CC studies of chorioretinal diseases, such as decorrelation signal indices, signal binarization, and analysis of signal voids. For calculating the CC decorrelation signal index, OCTA CC images were converted into grayscale values, attributing each pixel a value that represents the strength of the decorrelation signal. The CC decorrelation signal index was defined as the average decorrelation value of all pixels in the en face CC angiogram [28]. Binarization of OCTA images was described by Nicolò et al. [32] using the Otsu method, which is an automatic threshold selection from 
gray-level histograms. Binarization relies on conversion of a "noised" image in a white/black dichotomy, applying an automatic threshold to the original grayscale. The CC vascular flow area was defined as the percentage of the portion of white pixels against the whole scan area. Spaide $[33,34]$ proposed for the first time not to analyze areas of positive decorrelation signals, but to look at signal voids instead. According to his approach, automatic local thresholding was performed with the Phansalkar method. All thresholded areas $\geq 1$ pixel showing no flow information were measured. The proportion of absent flow signal accounted for by signal voids $>10,000 \mathrm{~mm}^{2}$ and signal voids $>40,000 \mathrm{~mm}^{2}$ were evaluated.

Another interesting approach to CC flow quantification was reported by Kurokawa et al. [35], who performed a CC study based on an adaptive optics OCTA (AOOCTA) prototype visualizing and quantifying the morphometry of capillaries in the CC in the living human retina for the first time. Four morphometric parameters of CC can be evaluated with this technique: RPE to CC depth separation, capillary diameter, normalized capillary density, and capillary length per unit area. Unlike OCTA devices with a lateral resolution of $15-20 \mu \mathrm{m}, \mathrm{AO}-$ OCTA offers a lateral resolution at the CC layer of $4.9 \mu \mathrm{m}$ sufficient to resolve single CC vessels. The authors concluded that AO-OCTA allows for a more complete description of CC capillary morphometry than OCTA alone and is more consistent with histology.

A major limitation in CC imaging with OCTA is that the images have a limited capability to see the intervascular spaces. Recently, Uji et al. [36] presented a multiple en face averaging approach in healthy volunteers that markedly improves CC visualization on OCTA images, transforming the images from a granular appearance to a level where the intervascular spaces can be resolved. The morphologic patterns seen in averaged CC images closely mimic the meshwork pattern observed on CC histology. Thus, this approach potentially allows for more precise quantitative metrics to be generated, such as CC vessel caliber.

\section{OCTA in Healthy Subjects}

In OCTA imaging, the CC appears as a mesh-like homogeneous layer whose individual vessels are usually not discernible. Visualization of flow in individual CC vessels is below the resolution limit of current OCTA devices, yet areas of absent flow signal, called signal voids, are resolvable and analyzable, as proposed by Spaide [33]. Monte- sano et al. [37] performed an in depth analysis of the structural features of normal CC imaged with OCTA and developed an extensive characterization of the normal subfoveal CC that can be used for flow analysis and investigation of pathologic alterations.

Data on the correlation of CC measurements and axial length in healthy subjects are ambiguous, although one may expect that the density of the vascular layers decreases with increasing axial length. In a study by Wang et al. [38] including 105 healthy participants with an axial length between 21.44 and $28.16 \mathrm{~mm}$, CC vessel density was not significantly correlated with axial length. In another study by Al-Sheikh et al. [39] including 28 patients with an axial length $>26.5 \mathrm{~mm}$, OCTA demonstrated an increase in the total and average area of flow voids in the CC. Whether the reduced CC perfusion may be the result of a stretching of the CC occurring with increased axial enlargement or whether this must rather be regarded as a pathologic change in terms of a choroidal loss needs to be investigated in future studies.

In a study of 36 healthy subjects ranging from 23 to 87 years of age, two different OCTA devices concordantly proved a correlation between advancing age and decreasing CC decorrelation signal, which is in accordance with previous histologic studies [13].

\section{OCTA in Dry AMD}

The molecular mechanisms involved in dry AMD pathogenesis are not fully understood. Numerous studies revealed evidence for a mutualistic relationship between the RPE and the CC. The production of trophic factors, including vascular endothelial growth factor (VEGF) by the RPE, ensures CC survival, while CC atrophy results in tissue hypoxia accelerating RPE and photoreceptor dysfunction [5]. A recent histopathologic study by Biesemeier et al. [7] suggests that changes in CC precede the degeneration of the RPE in AMD. Whether alterations in the CC are in fact the primary event in nonexudative AMD or secondary to molecular changes in the RPE is still a matter of debate. Notably, in vivo RPE function is currently not accessible to imaging, whereas decreased CC perfusion is indeed measurable in OCTA. Spaide [33] showed decreased CC perfusion manifested by decreased flow signal and large signal voids.

In neovascular AMD, Moult et al. [26] found regions of CC alterations either corresponding to flow impairment and/or atrophy adjacent to the choroidal neovascularization $(\mathrm{CNV})$. These findings suggest that $\mathrm{CNV}$ de- 
velops in areas of CC flow alteration to compensate for the reduced circulation. In 2012, Bhutto and Lutty [3] hypothesized that CC loss causes ischemia that makes the RPE release vasogenic factors, including VEGF, resulting in CNV development.

\section{OCTA in Early/Intermediate AMD}

Our group reported for the first time significantly reduced CC vessel density and reduced CC flow in eyes with reticular pseudodrusen (RPD) compared with healthy control eyes, suggesting a CC functional compromise in RPD eyes [28]. Later, Nesper et al. [40] confirmed these results using an OCTA metric for CC nonperfusion. They found that RPD eyes had significantly larger nonperfused areas compared with eyes with drusen without RPD. Additionally, the percentage of nonperfused CC on OCTA correlated with visual acuity in RPD eyes. Similar results were reported by Cicinelli et al. [41] by means of binarized OCTA scans. Chatziralli et al. [42] additionally observed that one-third of patients with RPD also revealed CC flow impairment in areas outside of RPD affection.

Presence of nonperfusion of the CC on OCTA images may be caused by CC flow velocities below the decorrelation threshold, a reduced number of CC vessels per unit area, a decreased CC vessel caliber, or completely obliterated capillaries. Either way, these OCTA CC findings suggest a pivotal role of the CC in RPD pathogenesis. The significant CC compromise in RPD eyes supports the notion that subretinal drusenoid deposit accumulation on the apical side of the RPE cells is a nonspecific consequence of outer retina hypoxia.

There is evidence indicating that the site of soft drusen formation is primarily caused by vascular dynamics. Drusen are prone to form at sites of insufficient choroidal perfusion, acting as a surrogate marker for CC dysfunction [43]. Soft drusen attenuate the OCT signal and cause artifacts in the underlying CC (Fig. 2), which was interpreted as a false-positive flow impairment by different authors $[17,44,45]$. Recently, a high rate of signal attenuation in CC slabs caused by soft drusen as well as segmentation errors in the proximity of drusen were reported using SD-OCTA. If shadowing artifacts in the structural en face OCT CC images are present, neither a qualitative nor a quantitative flow evaluation under drusen in CC OCTA is valid. Both signal attenuation in CC slabs and segmentation errors are frequent artifacts in SD-OCTA imaging in patients with soft drusen and must be considered during image analysis [22]. Lane et al. [45] compared the effect of shadowing in SD-OCTA with a noncommer-

Imaging the Choriocapillaris Using OCTA cial SS-OCTA prototype and concluded that 1,050-nm SS-OCTA is less vulnerable to producing areas of falsepositive flow impairment under drusen. Borrelli et al. [46] found that intermediate AMD eyes of patients with neovascular AMD in the fellow eye have an increased average CC signal void size compared to eyes of patients with intermediate AMD without neovascular AMD in the fellow eye. They suggest that CC signal void size may serve as a useful parameter for evaluating eyes with AMD in the future.

\section{OCTA in GA}

GA is a field where CC OCTA may contribute further insights into the underlying pathology. In a study of patients with GA due to AMD using an OCTA prototype in combination with the VISTA algorithm, Choi et al. [27] found that CC alterations can be detected beyond the GA margin. CC flow impairment outside the regions of GA was less pronounced than flow impairment occurring within the GA regions. Interestingly, a recent study by Kvanta et al. [47] consistently reported that CC flow outside the GA area with preserved RPE or in transitional areas was also impaired. Another study by Moult et al. [48] found focal regions of CC flow alteration underlying nascent GA and drusen-associated GA as well as a diffuse $\mathrm{CC}$ flow impairment throughout the imaged field. These preliminary results underscore the theory that CC breakdown precedes RPE and photoreceptor degeneration [7]. CC flow impairment at the margin of GA may also be a potential predictor of GA growth with regards to both growth direction and growth rate [49]. Possibly, the extent of CC flow impairment correlates with different hyperautofluorescent patterns at the margin of GA [50]. Sacconi et al. [51] reported a distinct CC vessel density decrease at the GA margin, while fundus autofluorescence did not reveal atrophic RPE at the same location. The authors suggest that their data support the theory that the CC damage precedes RPE loss in GA. The same group proposes to use OCTA as a tool to quantify GA areas [52].

A recent report by Pellegrini et al. [53] comparing CC alterations in GA and Stargardt disease using OCTA shed light on the differences in pathogenesis between both diseases. Stargardt patients revealed an extensive loss of CC with persisting tissue at its margins, whereas in patients with GA, the area of RPE loss showed persistent but distinctly rarefied CC vasculature.

The concurrence of anti-VEGF agents and the introduction of refined OCT devices paved the way for a successful treatment of neovascular AMD 10 years ago. With 

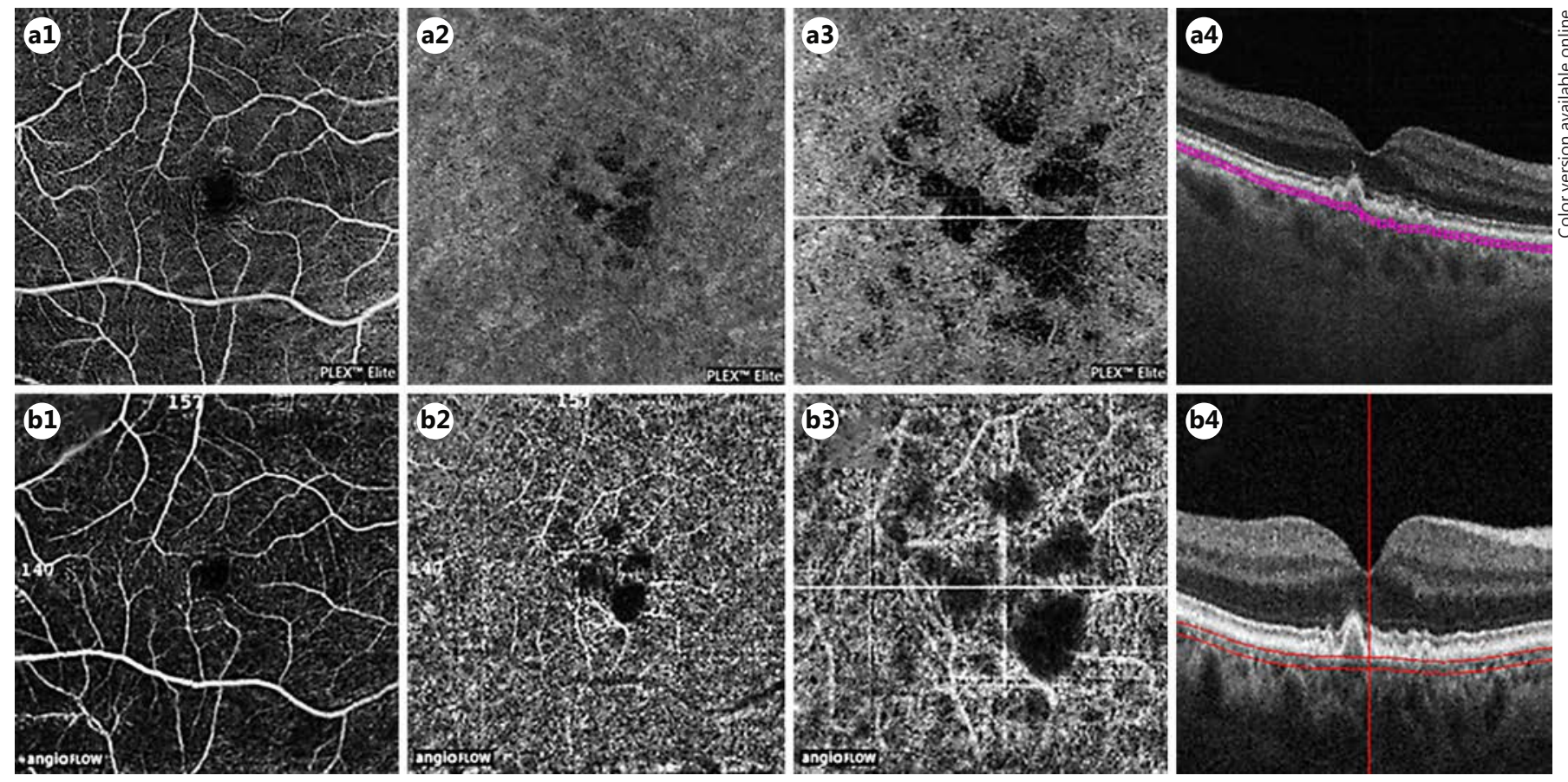

Fig. 2. Patient (79 years, female) with drusen due to early age-related macular degeneration imaged with a swept-source optical coherence tomography angiography (OCTA) device (Zeiss PLEX Elite 9000, a1-a4) and with a spectral-domain OCTA device (Optovue AngioVue, b1-b4). a1, b1 A $6 \times 6 \mathrm{~mm}^{2}$ en face image with standard segmentation of the superficial vascular layer without any abnormalities. a2, b2 A $6 \times 6 \mathrm{~mm}^{2}$ en face image with choriocapillaris (CC) standard segmentation showing signal voids due to

shadowing artifacts caused by soft drusen. a3, b3 A $3 \times 3 \mathrm{~mm}^{2}$ en face image with CC standard segmentation showing the area of interest. The horizontal white line indicates the position of the B-scan shown below. Projection artifacts of retinal vessels can be observed more clearly in b3, while signal voids caused by drusen appear to be slightly stronger expressed in a3. a4, b4 Corresponding B-scan showing subfoveal soft drusen under the retinal pigment epithelium.

the advent of selective CC imaging in OCTA and promising innovative phase III drugs under way, a similar scenario may hopefully evolve for the treatment of dry AMD.

\section{OCTA in CSC}

CSC defines a condition of serous neurosensory retinal detachment at the posterior pole resulting from defects in the RPE and leakage from the choroid occasionally associated with PED [54]. Most patients have a good visual prognosis with spontaneous resolution of subneurosensory fluid. However, visual outcome can be compromised due to a recurrent course of the disease or due to development of secondary neovascular membranes. The pathophysiology of CSC is often attributed to the choroid/CC, but the exact mechanism remains unknown. In the past, changes in choroidal blood flow have already been reported in CSC patients based on laser Doppler flowmetry, laser interferometry, and laser speckle flow-

graphy. Besides, areas of nonperfused CC seen during ICGA were described [55-57]. Interestingly, isometric exercise induced a more significant increase in choroidal blood flow in chronic CSC patients than in healthy patients, suggesting an inadequate vasoconstrictor response [58]. Using laser speckle flowgraphy, Saito et al. [59] recently correlated choroidal thickening with a decline in choroidal circulation in patients with CSC during spontaneous regression. The authors suggest that choroidal hyperperfusion triggers the elevation of hydrostatic pressure in the choroid, eventually followed by choroidal thickening at the acute phase of CSC.

Despite tremendous efforts using various therapeutic approaches such as photodynamic therapy, laser coagulation, anti-VEGF therapy, or mineralocorticoid receptor antagonists, there is no convincing treatment designed for CSC so far [60].

With the advent of OCTA, there is a new possibility to analyze vascular changes selectively at the level of the CC in CSC patients (Fig. 3). This new technology may con-

80

Ophthalmologica 2018;239:74-84

DOI: $10.1159 / 000485261$
Lauermann/Eter/Alten 
Fig. 3. Changes in choriocapillaris (CC) imaged with a swept-source optical coherence tomography angiography (OCTA) device (Zeiss PLEX Elite 9000, a) and with a spectral-domain OCTA device (Optovue AngioVue, b) in a patient (46 years, male) with chronic central serous chorioretinopathy for 10 months. a1, b1 A $6 \times 6 \mathrm{~mm}^{2}$ en face image with standard segmentation of the superficial vascular layer without any abnormalities. a2, b2 A $6 \times 6 \mathrm{~mm}^{2}$ en face image with standard CC segmentation showing irregular flow patterns (white circle). The horizontal white line indicates the position of the B-scan shown on the right. a3, b3 Corresponding B-scan showing subretinal fluid and abnormal dilatation of choroidal vessels (white arrow).
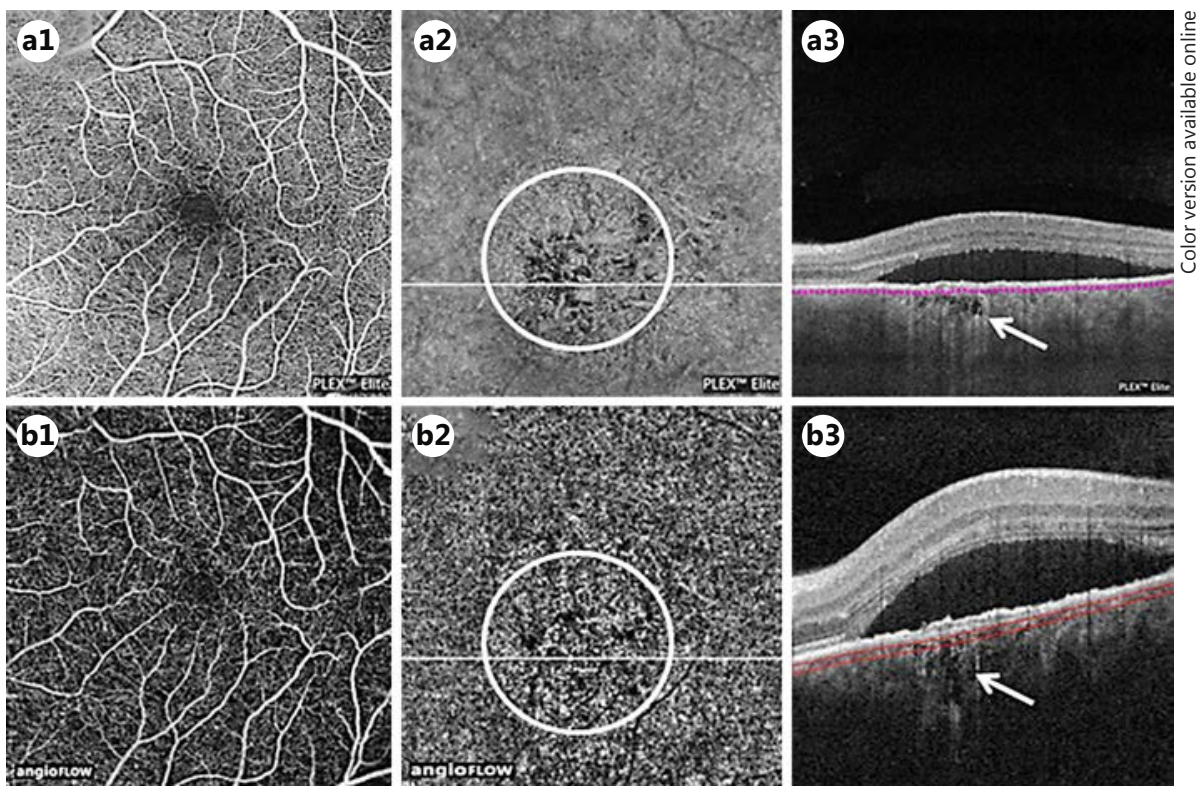

tribute to fully grasp the pathogenesis in the near future and to refine therapeutic options. Using OCTA, Costanzo et al. [61] systematically described multiple anomalies of the CC layer associated with CSC such as the presence of dark areas, dark spots, and dark vessels. The authors concluded that there was an association between dark areas and serous retinal detachment. Furthermore, dark spots were associated with RPE detachment and abnormal vessels were interpreted as asymmetric CNV. The authors interpreted abnormal choroidal patterns in OCTA as pathologically altered choroidal vasculature in CSC. These findings were often linked to CNV. Irregular CC flow patterns in OCTA corresponding to abnormalities seen in ICGA were also described by Teussink et al. [62]. Chan et al. [63] observed pathologically altered patterns in eyes with CSC as well. More than $80 \%$ of the CSC-affected eyes presented dilated vessels in the CC. This finding was associated with dye leakage in FA or hyperpermeability in ICGA. Feucht et al. [64] compared findings in OCTA and multimodal imaging in CSC patients. They could not identify leakage points in acute CSC using OCTA, but also observed general changes in CC flow. Bonini Filho et al. [65] compared the sensitivity and specificity of FA and OCTA with focus on CNV detection in chronic CSC and found a comparable sensitivity and specificity for the use of OCTA and coregistered OCT B-scans compared to FA. Nicolò et al. [32] examined quantitative changes of choroidal flow areas using SSOCTA. Interestingly, in that study, dark patterns of the

Imaging the Choriocapillaris Using OCTA choroid were present regardless of the amount of fluid in CSC as well as in fellow eyes, but such alterations were not present in healthy eyes. The choroidal vascular flow area was larger in CSC eyes compared with control eyes. At the same time, Nicolò et al. [32] demonstrated differences in flow areas between CC and deeper choroidal levels in eyes with CSC, possibly a result of compensatory flow mechanisms within the choroid/CC.

$\mathrm{Xu}$ et al. [66] evaluated the effects of half-dose PDT on CC patterns seen in OCTA. Notably, abnormal CC flow was detectable in all included eyes at baseline. At month 3 after PDT, the CC OCTA pattern had returned to normal in 97\%, supporting the efficacy of PDT and the potential diagnostic role of OCTA in CSC.

\section{Future Directions}

Further progress in different subfields of OCTA imaging appears necessary to fulfill the high expectations and hopes in this technology. The following aspects apply to OCTA in general as well as to OCTA CC imaging in particular.

So far, vascular depth discrimination has been limited by superficial vessels projecting flow signal artifacts onto deeper layers, e.g., prominent shadowing artifacts of retinal vessels visible in the CC en face slab. Campbell et al. [67] recently described a projection-resolved OCTA algorithm that improves depth resolution by removing 
projection artifacts while retaining in situ flow signal from real blood vessels in deeper layers. VISTA is another already mentioned algorithm to quantify relative blood flows which is particularly suitable for measuring slow CC flow [27]. Smoothed and resolved thresholding (SmART) display is a new OCTA display technique to resolve the ambiguity between areas of low OCT signal and areas of low blood flow [68]. This approach has the potential to reduce misinterpretation of OCTA images.

Currently available OCTA devices have a relatively poor axial resolution $(\sim 15 \mu \mathrm{m})$ due to signal averaging, which limits the identification of small-caliber vessels and is particularly relevant in imaging CC. Second-generation OCTA devices will certainly offer higher scanning speeds and larger fields of view with higher resolution and a decrease in artifacts from motion contrast. Applicability of higher-wavelength light will also enable better resolution of deeper structures. OCTA manufacturers must realize that adherence to certain standards in OCTA hardware and software will greatly facilitate the use of this technology in clinical science.

In general, the understanding of the newly developed imaging technology of OCTA is still in its nascent stages. Its application to the $\mathrm{CC}$ provides several challenges to overcome, but at the same time shows great promise as a powerful new approach to investigate the $\mathrm{CC}$ in various chorioretinal diseases. Reaching the diagnostic specificity and sensitivity of conventional angiography and warranting high reproducibility are important steps to establish this new imaging modality. Certainly, more studies are required in larger cohorts to further establish the possible association between CC flow compromise and chorioretinal pathology not only in dry AMD and CSC. Currently, OCTA for CC imaging has its place in a research setting or as a part of a multimodal imaging approach. In the future, quantitative parameters of CC perfusion may be used as possible clinical endpoints in observational and interventional studies. In the future, $\mathrm{CC}$ flow alterations might serve as an early diagnostic parameter in different chorioretinal diseases prior to the development of clinical signs.

\section{Disclosure Statement}

J.L. Lauermann: Bayer, Novartis. N. Eter: Novartis, Bayer, Pfizer, Roche, Allergan. F. Alten: Bayer. All authors certify that they have no affiliations with or involvement in any organization or entity with any financial interest (such as honoraria; educational grants; participation in speakers' bureaus; membership, employment, consultancies, stock ownership, or other equity interest; and expert testimony or patent licensing arrangements) or nonfinancial interest (such as personal or professional relationships, affiliations, knowledge or beliefs) in the subject matter or materials discussed in this paper. No funding was received for this research.

\section{References}

1 Fryczkowski AW: Anatomical and functional choroidal lobuli. Int Ophthalmol 1994;18: 131-141.

2 Hayreh SS: The choriocapillaris. Albrecht von Graefes Arch Klin Exp Ophthalmol 1974;192: 165-179.

3 Bhutto I, Lutty G: Understanding age-related macular degeneration (AMD): relationships between the photoreceptor/retinal pigment epithelium/Bruch's membrane/choriocapillaris complex. Mol Aspects Med 2012;33:295317.

4 Ramrattan RS, van der Schaft TL, Mooy CM, de Bruijn WC, Mulder PG, de Jong PT: Morphometric analysis of Bruch's membrane, the choriocapillaris, and the choroid in aging. Invest Ophthalmol Vis Sci 1994;35:2857-2864.

5 McLeod DS, Grebe R, Bhutto I, Merges C, Baba T, Lutty GA: Relationship between RPE and choriocapillaris in age-related macular degeneration. Invest Ophthalmol Vis Sci 2009;50:4982-4991.
6 Mullins RE, Johnson MN, Faidley EA, Skeie JM, Huang JA: Choriocapillaris vascular dropout related to density of drusen in human eyes with early age-related macular degeneration. Invest Ophthalmol Vis Sci 2011;52: 1606-1612.

7 Biesemeier A, Taubitz T, Julien S, Yoeruek E, Schraermeyer U: Choriocapillaris breakdown precedes retinal degeneration in age-related macular degeneration. Neurobiol Aging 2014;35:2562-2573.

8 Guyer DR, Yannuzzi LA, Slakter JS, Sorenson JA, Ho A, Orlock D: Digital indocyanine green videoangiography of central serous chorioretinopathy. Arch Ophthalmol 1994; 112:1057-1062.

9 Jia Y, Bailey ST, Hwang TS, McClintic SM, Gao SS, Pennesi ME, Flaxel CJ, Lauer AK, Wilson DJ, Hornegger J, Fujimoto JG, Huang D: Quantitative optical coherence tomography angiography of vascular abnormalities in the living human eye. Proc Natl Acad Sci USA 2015;112:E2395-E2402.
10 Alnawaiseh M, Rosentreter A, Hillmann A, Alex AF, Niekämper D, Heiduschka P, Pap T, Eter N: OCT angiography in the mouse: a novel evaluation method for vascular pathologies of the mouse retina. Exp Eye Res 2016; 145:417-423.

11 Sambhav K, Grover S, Chalam KV: The application of optical coherence tomography angiography in retinal diseases. Surv Ophthalmol 2017;62:838-866.

12 Munk MR, Giannakaki-Zimmermann H, Berger L, Huf W, Ebneter A, Wolf S, Zinkernagel MS: OCT-angiography: a qualitative and quantitative comparison of 4 OCT-A devices. PLoS One 2017;12:e0177059.

13 Lauermann JL, Heiduschka P, Nelis P, Treder $\mathrm{M}$, Alnawaiseh M, Clemens CR, Eter N, Alten F: Comparison of choriocapillaris flow measurements between two optical coherence tomography angiography devices. Ophthalmologica 2017;237:238-246. 
14 Potsaid B, Baumann B, Huang D, Barry S, Cable AE, Schuman JS, Duker JS, Fujimoto JG: Ultrahigh speed $1050 \mathrm{~nm}$ swept source/Fourier domain OCT retinal and anterior segment imaging at 100,000 to 400,000 axial scans per second. Opt Express 2010;18: 20029-20048.

15 Miller AR, Roisman L, Zhang Q, Zheng F, Rafael de Oliveira Dias J, Yehoshua Z, Schaal KB, Feuer W, Gregori G, Chu Z, Chen CL, Kubach S, An L, Stetson PF, Durbin MK, Wang RK, Rosenfeld PJ: Comparison between spectraldomain and swept-source optical coherence tomography angiographic imaging of choroidal neovascularization. Invest Ophthalmol Vis Sci 2017;58:1499-1505.

16 Su GL, Baughman DM, Zhang Q, Rezaei K, Lee AY, Lee CS: Comparison of retina specialist preferences regarding spectral-domain and swept-source optical coherence tomography angiography. Clin Ophthalmol 2017;11: 889-895.

17 Spaide RF, Fujimoto JG, Waheed NK: Image artifacts in optical coherence tomography angiography. Retina 2015;35:2163-2180.

18 Chen FK, Viljoen RD, Bukowska DM: Classification of image artefacts in optical coherence tomography angiography of the choroid in macular diseases. Clin Exp Ophthalmol 2016;44:388-399.

19 Lauermann JL, Treder M, Heiduschka P, Clemens CR, Eter N, Alten F: Impact of eyetracking technology on OCT-angiography imaging quality in age-related macular degeneration. Graefes Arch Clin Exp Ophthalmol 2017;255:1535-1542.

20 Ghasemi Falavarjani K, Al-Sheikh M, Akil H, Sadda SR: Image artefacts in swept-source optical coherence tomography angiography. $\mathrm{Br}$ J Ophthalmol 2017;101:564-568.

21 Niu S, Chen Q, de Sisternes L, Rubin DL, Zhang W, Liu Q: Automated retinal layers segmentation in SD-OCT images using dual gradient and spatial correlation smoothness constraint. Comput Biol Med 2014;54:116128.

22 Alten F, Lauermann JL, Clemens CR, Heiduschka P, Eter N: Signal reduction in choriocapillaris and segmentation errors in spectral domain OCT angiography caused by soft drusen. Graefes Arch Clin Exp Ophthalmol 2017; 255:2347-2355.

23 Louzada RN, de Carlo TE, Adhi M, Novais EA, Durbin MK, Cole E, Lane M, Moghimi O, Arya M, Filho MB, Witkin AJ, Baumal CR, Ávila M, Duker JS, Waheed NK: Optical coherence tomography angiography artifacts in retinal pigment epithelial detachment. Can J Ophthalmol 2017;52:419-424.

24 Veronese C, Maiolo C, Morara M, Armstrong GW, Ciardella AP: Optical coherence tomography angiography to assess pigment epithelial detachment. Retina 2016;36:645-650.
25 Rahimy E, Freund KB, Larsen M, Spaide RF, Costa RA, Hoang Q, Christakopoulos C, Munch IC, Sarraf D: Multilayered pigment epithelial detachment in neovascular age-related macular degeneration. Retina 2014;34: 1289-1295.

26 Moult E, Choi W, Waheed NK, Adhi M, Lee B, Lu CD, Jayaraman V, Potsaid B, Rosenfeld PJ, Duker JS, Fujimoto JG: Ultrahigh-speed swept-source OCT angiography in exudative AMD. Ophthalmic Surg Lasers Imaging Retina 2014;45:496-505.

27 Choi W, Moult EM, Waheed NK, Adhi M, Lee B, Lu CD, de Carlo TE, Jayaraman V, Rosenfeld PJ, Duker JS, Fujimoto JG: Ultrahigh-speed, swept-source optical coherence tomography angiography in nonexudative age-related macular degeneration with geographic atrophy. Ophthalmology 2015;122: 2532-2544

28 Alten F, Heiduschka P, Clemens CR, Eter $\mathrm{N}$ : Exploring choriocapillaris under reticular pseudodrusen using OCT-angiography. Graefes Arch Clin Exp Ophthalmol 2016;254: 2165-2173.

29 Wajer SD, Taomoto M, McLeod DS, McCally RL, Nishiwaki H, Fabry ME, Nagel RL, Lutty GA: Velocity measurements of normal and sickle red blood cells in the rat retinal and choroidal vasculatures. Microvasc Res 2000; 60:281-293.

30 Ferrara D, Waheed NK, Duker JS: Investigating the choriocapillaris and choroidal vasculature with new optical coherence tomography technologies. Prog Retin Eye Res 2016;52: 130-155.

31 Ploner SB, Moult EM, Choi W, Waheed NK, Lee B, Novais EA, Cole ED, Potsaid B, Husvogt L, Schottenhamml J, Maier A, Rosenfeld PJ, Duker JS, Hornegger J, Fujimoto JG: Towards quantitative optical coherence tomography angiography: visualizing blood flow speeds in ocular pathology using variable interscan time analysis. Retina 2016;36(suppl 1):S118-S126.

32 Nicolò M, Rosa R, Musetti D, Musolino M, Saccheggiani M, Traverso CE: Choroidal vascular flow area in central serous chorioretinopathy using swept-source optical coherence tomography angiography. Invest Ophthalmol Vis Sci 2017;58:2002-2010.

33 Spaide RF: Choriocapillaris flow features follow a power law distribution: implications for characterization and mechanisms of disease progression. Am J Ophthalmol 2016;170:5867.

34 Spaide RF: Choriocapillaris signal voids in maternally inherited diabetes and deafness and in pseudoxanthoma elasticum. Retina 2017;37:2008-2014.

35 Kurokawa K, Liu Z, Miller DT: Adaptive optics optical coherence tomography angiography for morphometric analysis of choriocapillaris [Invited]. Biomed Opt Express 2017;8: 1803-1822.
36 Uji A, Balasubramanian S, Lei J, Baghdasaryan E, Al-Sheikh M, Sadda SR: Choriocapillaris imaging using multiple en face optical coherence tomography angiography image averaging.JAMAOphthalmol2017;135:1197-1204.

37 Montesano G, Allegrini D, Colombo L, Rossetti LM, Pece A: Features of the normal choriocapillaris with OCT-angiography: density estimation and textural properties. PLoS One 2017;12:e0185256.

38 Wang Q, Chan S, Yang JY, You B, Wang YX, Jonas JB, Wei WB: Vascular density in retina and choriocapillaris as measured by optical coherence tomography angiography. Am J Ophthalmol 2016;168:95-109.

39 Al-Sheikh M, Phasukkijwatana N, Dolz-Marco R, Rahimi M, Iafe NA, Freund KB, Sadda SR, Sarraf D: Quantitative OCT angiography of the retinal microvasculature and the choriocapillaris in myopic eyes. Invest Ophthalmol Vis Sci 2017;58:2063-2069.

40 Nesper PL, Soetikno BT, Fawzi AA: Choriocapillaris nonperfusion is associated with poor visual acuity in eyes with reticular pseudodrusen. Am J Ophthalmol 2017;174:42-55.

41 Cicinelli MV, Rabiolo A, Marchese A, de Vitis L, Carnevali A, Querques L, Bandello F, Querques G: Choroid morphometric analysis in non-neovascular age-related macular degeneration by means of optical coherence tomography angiography. Br J Ophthalmol 2017; 101:1193-1200.

42 Chatziralli I, Theodossiadis G, Panagiotidis D, Pousoulidi P, Theodossiadis P: Choriocapillaris' alterations in the presence of reticular pseudodrusen compared to drusen: study based on OCTA findings. Int Ophthalmol 2017, Epub ahead of print.

43 Lengyel I, Tufail A, Hosaini HA, Luthert P, Bird AC, Jeffery G: Association of drusen deposition with choroidal intercapillary pillars in the aging human eye. Invest Ophthalmol Vis Sci 2004;45:2886-2892.

44 Zheng F, Roisman L, Schaal KB, Miller AR, Robbins G, Gregori G, Rosenfeld PJ: Artifactual flow signals within drusen detected by OCT angiography. Ophthalmic Surg Lasers Imaging Retina 2016;47:517-522.

45 Lane M, Moult EM, Novais EA, Louzada RN, Cole ED, Lee B, Husvogt L, Keane PA, Denniston AK, Witkin AJ, Baumal CR, Fujimoto JG, Duker JS, Waheed NK: Visualizing the choriocapillaris under drusen: comparing 1050 -nm swept-source versus 840 -nm spectral-domain optical coherence tomography angiography. Invest Ophthalmol Vis Sci 2016; 57:OCT585-OCT590.

46 Borrelli E, Uji A, Sarraf D, Sadda SR: Alterations in the choriocapillaris in intermediate age-related macular degeneration. Invest Ophthalmol Vis Sci 2017;58:4792-4798.

47 Kvanta A, Casselholm de Salles M, Amrén U, Bartuma H: Optical coherence tomography angiography of the foveal microvasculature in geographic atrophy. Retina 2017;37:936-942. 
48 Moult EM, Waheed NK, Novais EA, Choi W, Lee B, Ploner SB, Cole ED, Louzada RN, Lu CD, Rosenfeld PJ, Duker JS, Fujimoto JG: Swept-source optical coherence tomography angiography reveals choriocapillaris alterations in eyes with nascent geographic atrophy and drusen-associated geographic atrophy. Retina 2016;36(suppl 1):S2-S11.

49 Lindner M, Böker A, Mauschitz MM, Göbel AP, Fimmers R, Brinkmann CK, SchmitzValckenberg S, Schmid M, Holz FG, Fleckenstein M; Fundus Autofluorescence in Age-Related Macular Degeneration Study Group: Directional kinetics of geographic atrophy progression in age-related macular degeneration with foveal sparing. Ophthalmology 2015;122:1356-1365.

50 Bindewald A, Schmitz-Valckenberg S, Jorzik JJ, Dolar-Szczasny J, Sieber H, Keilhauer C, Weinberger AW, Dithmar S, Pauleikhoff D, Mansmann U, Wolf S, Holz FG: Classification of abnormal fundus autofluorescence patterns in the junctional zone of geographic atrophy in patients with age related macular degeneration. Br J Ophthalmol 2005;89:874878.

51 Sacconi R, Corbelli E, Carnevali A, Querques L, Bandello F, Querques G: Optical coherence tomography angiography in geographic atrophy. Retina 2017, Epub ahead of print.

52 Corbelli E, Sacconi R, Rabiolo A, Mercuri S, Carnevali A, Querques L, Bandello F, Querques G: Optical coherence tomography angiography in the evaluation of geographic atrophy area extension. Invest Ophthalmol Vis Sci 2017;58:5201-5208.

53 Pellegrini $M$, Acquistapace A, Oldani M, Cereda MG, Giani A, Cozzi M, Staurenghi G: Dark atrophy: an optical coherence tomography angiography study. Ophthalmology 2016;123:1879-1886.
54 Daruich A, Matet A, Dirani A, Bousquet E, Zhao M, Farman N, Jaisser F, Behar-Cohen F: Central serous chorioretinopathy: recent findings and new physiopathology hypothesis. Prog Retin Eye Res 2015;48:82-118.

55 Kitaya N, Nagaoka T, Hikichi T, Sugawara R, Fukui K, Ishiko S, Yoshida A: Features of abnormal choroidal circulation in central serous chorioretinopathy. Br J Ophthalmol 2003;87: 709-712.

56 Saito M, Saito W, Hashimoto Y, Yoshizawa C, Fujiya A, Noda K, Ishida S: Macular choroidal blood flow velocity decreases with regression of acute central serous chorioretinopathy. $\mathrm{Br}$ J Ophthalmol 2013;97:775-780.

57 Tittl M, Polska E, Kircher K, Kruger A, Maar $\mathrm{N}$, Stur M, Schmetterer L: Topical fundus pulsation measurement in patients with active central serous chorioretinopathy. Arch Ophthalmol 2003;121:975-978.

58 Tittl M, Maar N, Polska E, Weigert G, Stur M, Schmetterer L: Choroidal hemodynamic changes during isometric exercise in patients with inactive central serous chorioretinopathy. Invest Ophthalmol Vis Sci 2005;46:47174721 .

59 Saito M, Noda K, Saito W, Ishida S: Relationship between choroidal blood flow velocity and choroidal thickness in patients with regression of acute central serous chorioretinopathy. Graefes Arch Clin Exp Ophthalmol 2017, Epub ahead of print.

60 Wong KH, Lau KP, Chhablani J, Tao Y, Li Q, Wong IY: Central serous chorioretinopathy: what we have learnt so far. Acta Ophthalmol 2016;94:321-325.

61 Costanzo E, Cohen SY, Miere A, Querques G, Capuano V, Semoun O, El Ameen A, Oubraham H, Souied EH: Optical coherence tomography angiography in central serous chorioretinopathy. J Ophthalmol 2015;2015:134783.
62 Teussink MM, Breukink MB, van Grinsven MJ, Hoyng CB, Klevering BJ, Boon CJ, de Jong EK, Theelen T: OCT angiography compared to fluorescein and indocyanine green angiography in chronic central serous chorioretinopathy. Invest Ophthalmol Vis Sci 2015; 56:5229-5237.

63 Chan SY, Wang Q, Wei WB, Jonas JB: Optical coherence tomography angiography in central serous chorioretinopathy. Retina 2016;36: 2051-2058.

64 Feucht N, Maier M, Lohmann CP, Reznicek $\mathrm{L}$ : OCT angiography findings in acute central serous chorioretinopathy. Ophthalmic Surg Lasers Imaging Retina 2016;47:322-327.

65 Bonini Filho MA, de Carlo TE, Ferrara D, Adhi M, Baumal CR, Witkin AJ, Reichel E, Duker JS, Waheed NK: Association of choroidal neovascularization and central serous chorioretinopathy with optical coherence tomography angiography. JAMA Ophthalmol 2015; 133:899-906.

66 Xu Y, Su Y, Li L, Qi H, Zheng H, Chen C: Effect of photodynamic therapy on optical coherence tomography angiography in eyes with chronic central serous chorioretinopathy. Ophthalmologica 2017;237:167-172.

67 Campbell JP, Zhang M, Hwang TS, Bailey ST, Wilson DJ, Jia Y, Huang D: Detailed vascular anatomy of the human retina by projectionresolved optical coherence tomography angiography. Sci Rep 2017;7:42201.

68 Yiu P, Moult EM, Ploner SB, Lee B, Husvogt L, Maier A, Spaide RF, Duker JS, Waheed NK, Fujimoto JG:Smoothed and Resolved Thresholding (SmaRT-) display: a new OCTA display technique to resolve the low flow ambiguity. Invest Ophthalmol Vis Sci 2017;58:653. 